\title{
Utilización de TIC en el ámbito educativo de la Ingeniería de Procesos de Fabricación
}

\section{ICT using in Manufacturing Process Engeenering educational field}

\author{
Sergio Martín-Béjar ${ }^{1}$, María Jesús Martín Sánchez², Francisco Javier Trujillo Vilches³ ${ }^{3}$ \\ Carolina Bermudo Gamboa ${ }^{4}$ \\ ${ }^{1}$ Departamento de Ingeniería Civil, de Materiales y Fabricación, Universidad de Málaga, España (smartinb@uma.es) \\ ${ }^{2}$ Departamento de Ingeniería Civil, de Materiales y Fabricación, Universidad de Málaga, España (mjmartin@uma.es) \\ ${ }^{3}$ Departamento de Ingeniería Civil, de Materiales y Fabricación, Universidad de Málaga, España (trujillov@uma.es) \\ ${ }^{4}$ Departamento de Ingeniería Civil, de Materiales y Fabricación, Universidad de Málaga, España (carbergam@uma.es)
}

Recibido el 21 de marzo de 2018; revisado el 1 de mayo de 2018; aceptado el 24 de septiembre de 2018; publicado el 1 de junio de 2019

\section{RESUMEN:}

El uso de tecnologías de información y comunicación (TIC) se ha convertido en una herramienta que se utiliza cada día más en el ámbito educativo y en el ámbito laboral. Desde el punto de vista educativo, las TIC se han utilizado como herramientas facilitadoras del proceso de aprendizaje mientras que, desde el punto de vista laboral, se utiliza esta tecnología como medio fundamental para el control de procesos productivos como para el desarrollo de servicios.

Por ello, en la asignatura de Ingeniería de Fabricación de la Escuela de Ingeniería Industriales de la Universidad de Málaga, se ha propuesto al alumnado la realización de un trabajo para la edición de vídeos de tipo educativo que explique un proceso de fabricación.

Dicho trabajo se ha llevado a cabo en diferentes titulaciones donde se imparte la asignatura. Para ello se ha formado un equipo de trabajo por el personal docente de la asignatura, estableciendo una metodología para realizar el seguimiento y evaluación del trabajo realizado por el alumnado.

La elaboración de dicho trabajo ha originado la edición de 85 vídeos relacionados con la Ingeniería de los Procesos de Fabricación, los cuales pueden ser utilizados como recurso educativo en cursos posteriores y facilitar así la comprensión por parte del alumnado de los términos objeto de estudio.
PALABRAS
CLAVE:
RECURSOS EDUCATIVOS, VÍDEO EDUCATIVO, EDICIÓN, INGENIERÍA DE LA PRODUCCIÓN, TECNOLOGÍA DE LA INFORMACIÓN.

\begin{abstract}
:
Information and Communication Technologies (ICT) has become a very important tool in educational area and in workspace. From an educational point of view, ICT has been used as a learning process tools, while in work environment, these technologies has been used as services development and productive process control tools

Thus, in the Manufacturing Engineering subject at the Industrial Engineer Faculty of the University of Málaga, an educational vídeo edition that explains a term related to manufacturing engineering has been proposed to the students.

This work has been implemented in different grades where the subject is developed. Therefore, a teachers teamwork has been formed which established a tracking and evaluation methodology for the students vídeo elaboration.

The performance of this job has generated 85 vídeo editions of terms related with Manufacturing Process Engineering, which can be used as educational resources in future academic courses
\end{abstract}


KEYWORDS: EDUCATIONAL RESOURCES, EDUCATIONAL VÍDEO, EDITION, MANUFACTURING ENGINEERING, INFORMATION TECHNOLOGY.

\section{INTRODUCCIÓN}

Las TIC se han convertido en una herramienta fundamental en los procesos de aprendizaje, estableciendo una nueva metodología docente basada en la implementación de nuevos recursos educativos que, estando a disposición del alumnado, han facilitado el proceso de aprendizaje (Arias, 2008; Guerra, González y García, 2010, Morales, Trujillo y Raso, 2015, Hernández y Torrijos, 2019).

Es necesario considerar que, durante el periodo educativo, para el alumnado es el primer momento en el que comienza a utilizar estas herramientas. Con el avance tecnológico que se dispone hoy día, estas TIC se han convertido en herramientas de trabajo habituales, por lo que se considera necesario que el alumnado debe estar preparado en el uso de estas nuevas tecnologías para facilitar su posterior incorporación en el mercado laboral (Maquilón, Mirete, García y Hernández, 2013; Tello y Aguaded, 2009).

Son numerosos los medios tecnológicos utilizados a diario en el ejercicio de la profesión (diseño asistido por ordenador, fabricación asistida por ordenador, etc.), por lo que es necesario fomentar el uso de dichas herramientas en el ámbito de los estudios técnicos como los desarrollados en las escuelas de ingenierías y en las escuelas de arquitectura (Area, 2005; PrietoDíaz, 2011).

Cualquier actividad profesional suele formar equipos de trabajo multidisciplinar, ya que se consiguen alcanzar objetivos de menor dificultad. El trabajo en equipo requiere una colaboración organizada entre todos los integrantes y que cada uno de ellos aporte sus principales conocimientos o experiencias (Cardona y Wilkinson, 2006). Además, con la implantación de lo establecido en la Declaración de Bolonia, el aprendizaje colaborativo es un objetivo a tener en cuenta en el proceso de aprendizaje del alumnado (Cazorla, 2011), por lo que el trabajo en equipo se ha de tener en cuenta por parte del personal docente a la hora de desarrollar la actividad educativa.

En la asignatura de Ingeniería de Fabricación que se imparte en la Escuela de Ingenierías Industriales de la Universidad de Málaga, es habitual la utilización de vídeos como recurso educativo, en los que se muestra al alumnado diferentes procesos de fabricación, con objeto de que puedan familiarizarse con sus características técnicas así como con la tecnología utilizada en el proceso.

Por ello, durante el curso académico 2016-2017 se propuso al alumnado de Ingeniería de Fabricación, que desarrollase la edición de un vídeo relacionado con diferentes procesos o tecnologías que se desarrollan en la asignatura.

Esta actividad se ha propuesto a 4 grados y 3 dobles grados en los que se imparte la asignatura (tabla 1).

Tabla 1. Titulaciones en las que se imparte la asignatura

Grado en Ingeniería Mecánica (GIM)

Grado en Ingeniería Eléctrica (GIE)

Grado en Electrónica Industrial (GIEL)

Grado en Tecnologías Industriales (GITI)

Doble Grado en Ingeniería Mecánica y Eléctrica

Doble Grado en Ingeniería Eléctrica y Electrónica Industrial

Doble Grado en Ingeniería Mecánica y de Diseño Industrial y Desarrollo del producto

El alumnado de Doble Grado en Ingeniería Eléctrica y Electrónica Industrial y el Doble Grado en Ingeniería Mecánica y Eléctrica, se encuentra integrado en el aula con el alumnado perteneciente a la titulación de Grado en Ingeniería Eléctrica, por lo que en adelante se calificarán los grupos de estas titulaciones como alumnos de Grado en Ingeniería Eléctrica (GIE). De forma similar, los alumnos del Doble Grado en Ingeniería Mecánica y de Diseño Industrial y Desarrollo del producto, se encuentran integrados con los alumnos del Grado de Ingeniería Mecánica (GIM).

En cada grupo, se han formado equipos de trabajo formados por 3 o 4 integrantes y se les ha asignado un término relacionado con la asignatura, en la cual deberán realizar la edición de un vídeo que explique el término asignado.

Los términos propuestos están relacionados con diferentes ámbitos de la Ingeniería de Fabricación:

○ Fundición

- Pulvimetalurgia

- Procesado de polímeros y materiales compuestos

- Deformación plástica

- Soldadura

- Mecanizado

- Automatización de la fabricación

- Sistemas de fabricación

- Metrología

- Calidad

- Fabricación y medioambiente 
Una vez elaborado el vídeo sobre el término asignado, el resultado obtenido se ha expuesto en clase por el equipo de trabajo al resto del alumnado.

Además, el trabajo ha formado parte de la evaluación de la asignatura con una valoración del $10 \%$ de la nota final.

Para el correcto seguimiento y evaluación del trabajo propuesto se ha establecido una metodología que permite asegurar el cumplimiento de los requisitos establecidos en su elaboración, para lo cual se ha formado un grupo de trabajo compuesto por todo el personal docente que imparte la asignatura en todas las titulaciones anteriormente mencionadas.

También se ha propuesto la actividad formativa a un grupo de la titulación del Grado de Tecnologías Industriales que se imparte en inglés, debiendo realizar el alumnado el trabajo grupal en dicha lengua.

\section{LAS TIC COMO HERRAMIENTA EDUCATIVA}

El proceso de aprendizaje es una acción propia de cada individuo donde establece la complejidad del proceso desde una posición de conocimiento intuitiva y personal, también llamado como conocimiento primario. El desarrollo continuo de la sociedad genera la aparición de nuevos fenómenos emergentes que el individuo tiene que asimilar, de forma que es una oportunidad para el personal docente establecer estrategias que permitan relacionar nuevos fenómenos con conocimientos previos que el estudiante posee, bien sean innatos o personales, de forma que facilite el proceso de aprendizaje (Araya, 2017).

El uso de herramientas multimedia en el proceso de aprendizaje permite al alumnado asimilar los conocimientos de una forma más eficaz gracias a la aportación de información visual y auditiva en un único elemento (Mayer, 2005), donde la utilización de vídeos de tipo educativo, acompañado por la presencia de un docente, facilitará la adquisición de conocimiento, el aprendizaje percibido por el alumnado y establecer sinergias con otras metodologías de aprendizaje (Monedero, Higueras, y Luengo, 2017).

Establecer una metodología de trabajo focalizada en la participación de todos los integrantes de un grupo, metodología participativa, facilita la comunicación entre alumnado y docente, favorece la generación colaborativa de conocimiento y crea un entorno de aprendizaje colaborativo y aprendizaje informal (Antoli, 2015; Serrat y Jarauta, 2010).
El trabajo colaborativo también supone un recurso metodológico que contribuye a la calidad del proceso educativo que, además, genera un espacio capaz de crear actitudes y valores propios de una democracia, favoreciendo el reconocimiento y respeto de todos los integrantes del colectivo fomentando la inclusión (Río y Arias, 2015).

Además, la integración de las TIC en los procesos educativos y de investigación ayuda a los estudiantes, de forma individualizada, a desarrollar competencias; potencia la práctica de búsqueda, análisis, estructuración, adecuación y evaluación de la información; los capacita para solucionar problemas, tomar decisiones, fomentar la creatividad $\mathrm{y}$ eficacia en el uso de herramientas productivas, comunicadores, colaboradores, publicadores y productores, y ciudadanos informados, responsables y capaces de contribuir a la sociedad (Oswaldo y Gamonal, 2016).

Por tanto, la utilización de las TIC se ha convertido en una herramienta facilitadora del proceso de aprendizaje, la cual con el desarrollo tecnológico actual se encuentra accesible tanto al alumnado como al personal docente, por lo que se ha convertido en un medio indispensable para la mejora de la calidad educativa.

Numerosas son las experiencias que se han llevado a cabo en la integración de las TIC en diversos ámbitos educativos de titulaciones superiores.

Monedero, Higueras y Luengo (2017) realizaron diferentes vídeotutoriales sobre diferentes equipos que se encontraban en los laboratorios de audiovisuales, a los cuales colocaron diferentes códigos QR que permitían visualizar, mediante un dispositivo móvil que suele disponer el alumnado, el vídeotutorial con la explicación del manejo del equipo.

Ros y Rosa (2014) valoran de forma positiva la utilización de vídeos docentes como complemento formativo en una metodología innovadora como es la clase invertida o "inverted clasroom".

Ausín, Abella, Delgado y Hortigüela (2016) elaboran una radio educativa, donde crean diferentes podcast relacionados con las TIC creados a partir de grupos de trabajo colaborativo.

Quesada (2014) crea vídeos tutoriales como herramienta para la formación de futuros docentes de inglés evidenciando una motivación por parte del alumnado en el interés de la asignatura desarrollada.

De forma similar, Bustamante y colaboradores (2016) crean vídeos como píldoras formativas para estudiantes de magisterio en educación infantil que 
permiten una adquisición del aprendizaje de forma constructiva, significativa y activa.

Barroso, Cabero y Moreno (2016) evaluaron la influencia de la utilización de la realidad aumentada en la docencia de medicina, resultando un elemento motivador del alumnado dentro del aula, así como una herramienta que facilita el aprendizaje en un ámbito científico donde la actividad práctica en el desarrollo docente resulta ser bastante compleja.

Por tanto, la utilización de las TIC en la actividad docente resulta ser un elemento innovador, motivador y facilitador del proceso de aprendizaje por parte del alumnado.

\section{OBJETIVOS}

El objetivo principal del trabajo propuesto al alumnado en la asignatura de Ingeniería de Fabricación ha sido realizar la edición de un vídeo de tipo educativo relacionado con un término o concepto que se desarrolla en la asignatura. Dicho vídeo está formado por una selección de diferentes vídeos de libre uso, de donde seleccionan diferentes partes que consideran adecuadas para editar un nuevo vídeo que permita la explicación del término asignado en un tiempo no superior a 2 minutos.

Estos trabajos realizados se utilizan como base de datos para futuras consultas del alumnado o bien para su empleo como recurso durante la actividad docente de la asignatura en futuros cursos académicos.

En la elaboración del trabajo por parte del alumnado, también se han considerado un conjunto de objetivos secundarios:

- Conocer y utilizar diferentes programas de libre uso que permitan la edición de vídeos, dejando libertad de elección al grupo de trabajo.

- Buscar, catalogar y ordenar diferentes fuentes bibliográficas que sea de uso para el trabajo a desarrollar.

- Elaborar las referencias bibliográficas de forma correcta, relacionado con los recursos utilizados para la edición del vídeo.

- Realizar un breve informe donde se detalle cómo el grupo ha llevado a cabo el trabajo, identificando todos los recursos considerados y justificando su elección final.

- Fomentar el trabajo colaborativo entre el alumnado formando equipos por 3 o 4 personas.

- Comunicar en público el trabajo desarrollado por el grupo de trabajo.

\section{DISEÑO DE LA ACCIÓN DOCENTE}

Para la consecución de los objetivos establecidos, se han implementado dos metodologías, una de ellas orientada hacia el alumnado (figura 1), que es quien tiene que realizar el trabajo propuesto y una segunda, orientada hacia el personal docente, que permita establecer actuaciones para el seguimiento y evaluación de la actividad propuesta.

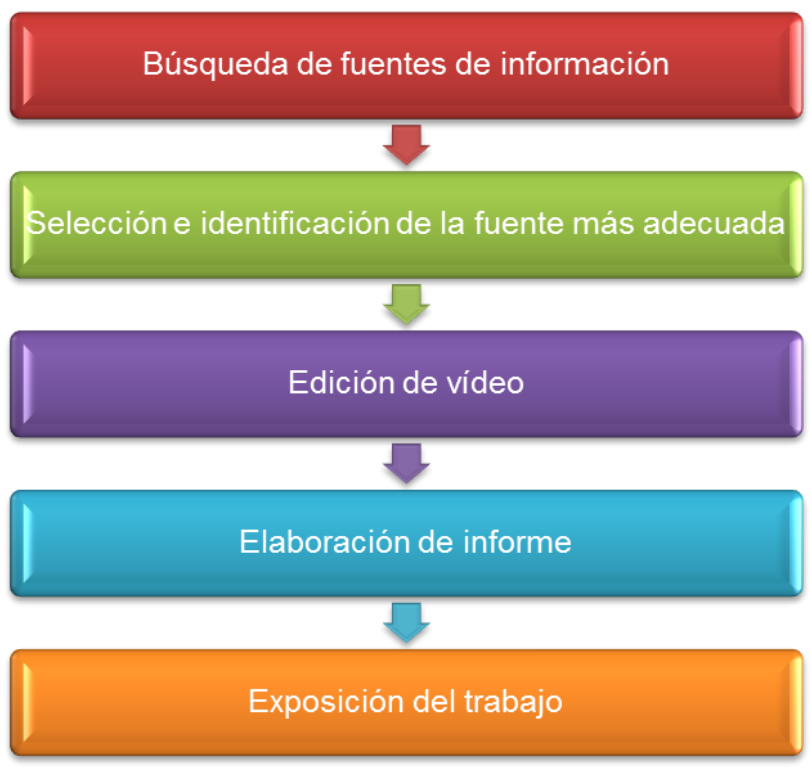

Figura 1. Propuesta metodológica para la elaboración del trabajo por parte del alumnado.

Al alumnado se le propone, a través del Campus Virtual de la Universidad de Málaga, un listado de términos correspondiente a cada uno de los trabajos a realizar, donde el alumnado, de forma individual se inscribe en el trabajo que desea realizar. En el momento que se completa un grupo con 4 integrantes, ya no será posible la inscripción, debiendo seleccionar un grupo donde aún queden huecos disponibles. En todo momento cada estudiante es conocedor del alumnado inscrito previamente en cada uno de los trabajos, permitiendo seleccionar el término así como el resto de integrantes que formarán parte del grupo de trabajo.

Asignado el término y formado el equipo de trabajo, se propone al alumnado que comience a trabajar buscando referencias bibliográficas relacionadas con el término a trabajar, para lo cual deben basar su búsqueda en fuentes de libre uso que se encuentran en internet.

Dichas fuentes bibliográficas corresponderán con imágenes o vídeos que permitan explicar las características de dicho proceso o término asignado. 
Evaluadas las referencias a utilizar, el alumnado seleccionará diferentes elementos con los que pueda editar un nuevo vídeo que realice una explicación correcta del proceso de fabricación. Para la edición del vídeo, el alumnado ha de utilizar software de libre uso (Avidemux, VirtualDub, etc.).

El equipo de trabajo consensua las referencias a utilizar y mediante el uso de software de edición de vídeo elabora un vídeo con una duración no superior a los 2 minutos.

Además de la edición del vídeo, el alumnado debe realizar un informe en el que se indican las actuaciones llevadas a cabo a lo largo de la ejecución del trabajo.

Ha de justificar cada una de las referencias utilizadas, indicando la aportación en el vídeo editado y se establece la bibliografía según norma IEEE (Ahmadi, 2011), siendo la norma habitualmente utilizada en el ámbito de la ingeniería.

Finalmente, el equipo de trabajo ha de exponer el resultado obtenido al resto de grupos de trabajo, para lo cual elabora una presentación que facilita dicha explicación, además de exponer el vídeo editado.

En referencia al personal docente, se ha establecido una metodología de trabajo que permite asegurar el seguimiento y evaluación de los trabajos propuestos al alumnado.

Inicialmente se elabora un listado de términos relacionados con la Ingeniería de los Procesos de Fabricación, los cuales se distribuyen entre el personal docente responsable de cada uno de los grupos de las diferentes titulaciones en las que se imparte la asignatura, de forma que se establece un registro de términos propuestos, evitando una repetición elevada de vídeos editados sobre un mismo término.

En el campus virtual se crea una actividad propia de Moodle, "Escoge grupo", por parte del docente responsable del grupo, que permite la inscripción del alumnado en el término en el que desean realizar el trabajo, limitando a un máximo de 4 integrantes en cada grupo de trabajo.

Para el seguimiento y evaluación de la actividad propuesta al alumnado, el profesorado realiza 5 sesiones en aula con una duración de 1,5 horas de cada una de ellas (figura 2).

En la primera sesión, el docente informa al alumnado de los objetivos y criterios de evaluación establecidos para la realización del trabajo. Se comunican los diferentes trabajos propuestos y se explica cómo ha de realizar la inscripción a través del Campus Virtual en el término que desean realizar.
En una segunda sesión en el aula, cada grupo indica las referencias bibliográficas seleccionadas inicialmente para la elaboración de los vídeos relacionados con el trabajo. Además, las dudas originadas en cada uno de los grupos de trabajo se exponen en el aula al resto de grupos, de forma que sirva para aportar la mayor información posible a todo el alumnado.

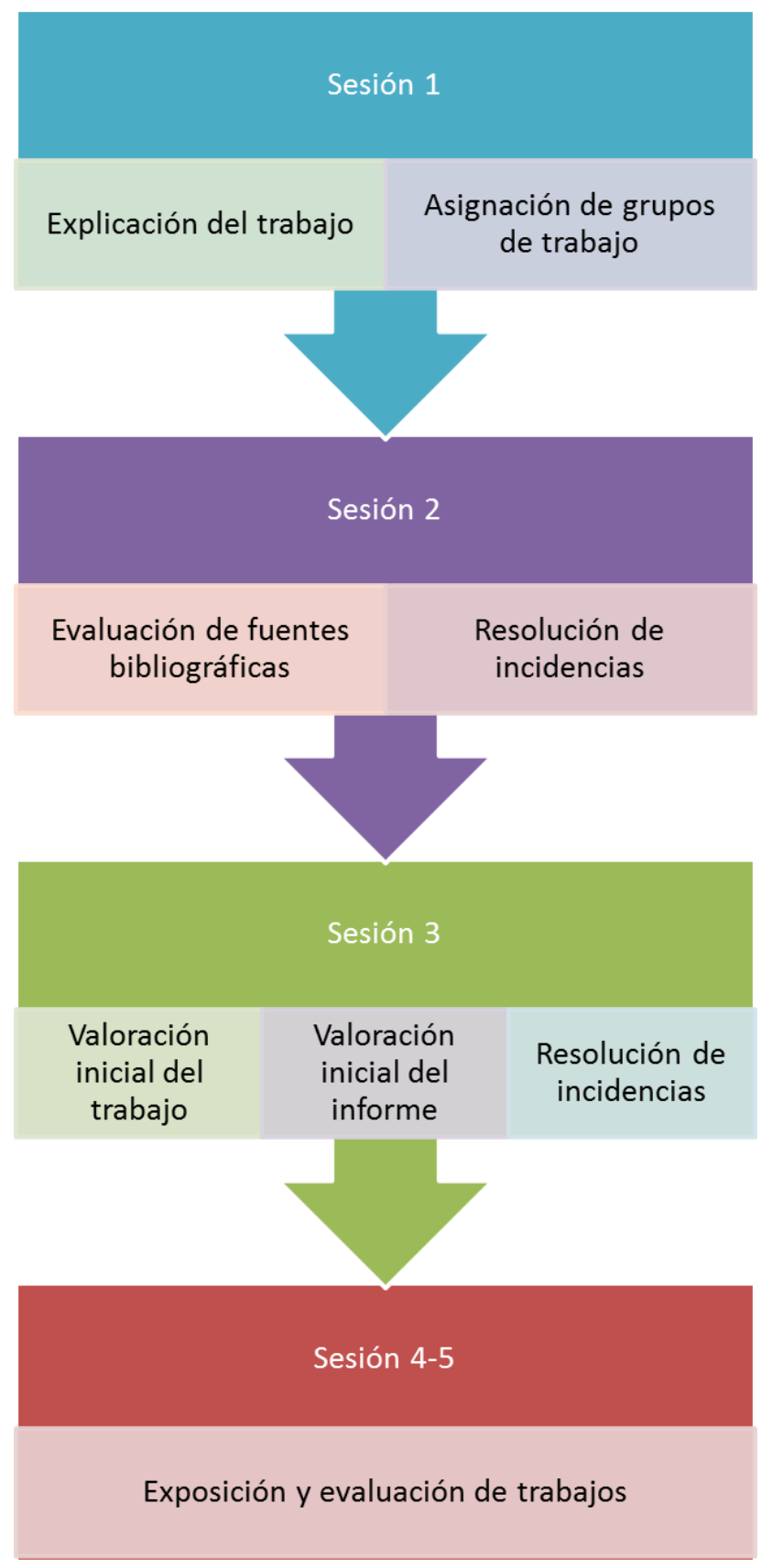

Figura 2. Sesiones para el seguimiento y evaluación de la actividad propuesta.

Avanzado el trabajo por parte del alumnado, se establece una tercera sesión que permite realizar una valoración inicial del trabajo, con objeto de corregir cualquier incidencia surgida, así como realizar una 
valoración inicial sobre el informe del trabajo. Además, se resuelven las dudas finales que propone cada grupo de trabajo, previa a la presentación definitiva del vídeo editado.

Finalmente, se realiza la exposición oral del trabajo realizado, así como la emisión del vídeo, por parte de cada uno de los grupos en dos sesiones presenciales en el aula. Para ello, un miembro del equipo de trabajo se encarga de la exposición, dejando finalmente un turno de preguntas para el tutor docente y el resto de los alumnos que se encuentran en el aula.

Para la evaluación del trabajo por el personal docente se establecen 6 criterios a considerar:

- Calidad del trabajo: considerando la originalidad del trabajo realizado, así como la correcta explicación del término asignado a través del vídeo editado.

- Adecuación a los requisitos: cumpliendo los plazos de entrega fijados inicialmente, tanto del informe justificativo del trabajo como del vídeo, así como del aspecto formal en la elaboración del informe y de la presentación para la exposición oral.

- Grado de dificultad de la búsqueda: valorando la dificultad del término a realizar por tratarse de procesos de fabricación innovadores cuya información disponible en internet o en otros medios de información como artículos o libros son menos numerosos.

- Calidad del informe justificativo del trabajo: evaluando la justificación de cada una de las referencias bibliográficas seleccionadas y la justificación de la organización de secuencias establecidas en el vídeo editado.

- Exposición oral: valorando la fluidez con la que el equipo de trabajo es capaz de exponer los resultados, así como de responder cuestiones realizadas por el profesorado o por el alumnado sobre el trabajo.

La cuantificación final de la evaluación del trabajo por el personal docente se establece con una valoración de 0 a 10 puntos.

\section{RESULTADO}

La metodología propuesta en el apartado anterior se ha aplicado durante el curso académico 2016-2017 en la asignatura de Ingeniería de Fabricación de diferentes titulaciones de grados de la Escuela de Ingenierías Industriales de la Universidad de Málaga como se ha comentado con anterioridad.
Para ello, un total de 8 docentes han tutorizado la realización de la actividad, que se ha llevado en los 10 grupos de docencia en los que se imparte la asignatura de Ingeniería de Fabricación.

Han participado en la realización del trabajo un total de 409 estudiantes, de todas las titulaciones, sobre un total de 460 estudiantes inscritos en la asignatura, lo que supone una participación del $89,51 \%$ (tabla 2 ).

Tabla 2. Participación de alumnado por titulaciones (\%)

\begin{tabular}{cc}
\hline Grado y Doble Grado & Participación (\%) \\
\hline GIE & 86,82 \\
GIM & 92,00 \\
GITI & 89,71 \\
GIEL & 82,22 \\
\hline
\end{tabular}

Se han constituido un total de 111 grupos de trabajo entre los estudiantes (tabla 3), a los cuales se les ha han asignado en total 85 términos diferentes, relacionados con la asignatura.

Tabla 3. Número de grupos de trabajo por titulaciones

\begin{tabular}{cc}
\hline Grado y Doble Grado & $\mathrm{N}^{\circ}$ grupos de trabajo \\
\hline GIE & 35 \\
GIM & 32 \\
GITI & 33 \\
GIEL & 11 \\
\hline
\end{tabular}

Se ha evaluado la totalidad de los trabajos bajos los criterios establecidos en la metodología de evaluación, habiéndose obtenido una nota media de 8,02 puntos sobre una evaluación de 10 puntos.

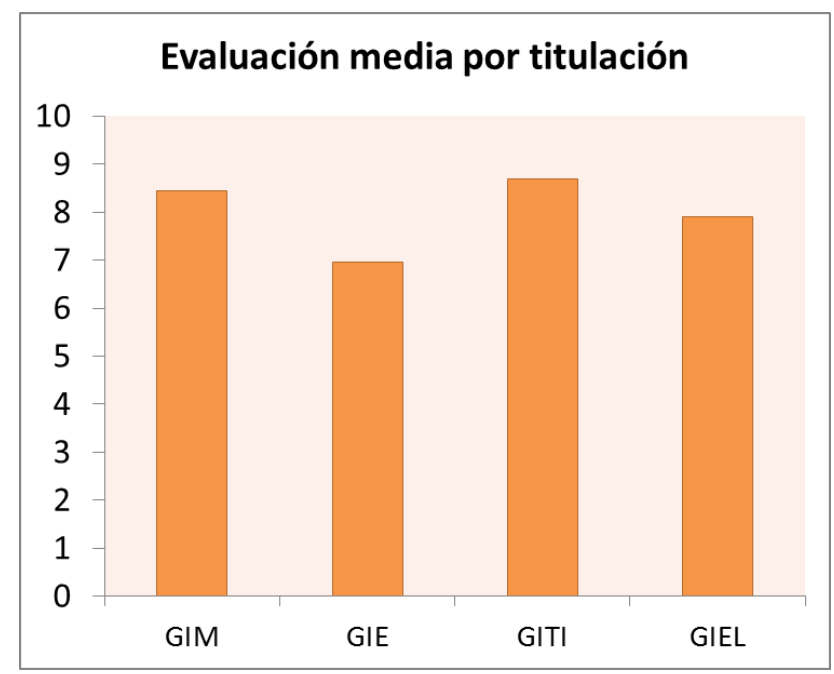

Figura 3. Evaluación media por titulación académica

Como se ha indicado anteriormente, en el grado en Ingeniería de Tecnologías Industriales existe un grupo de la asignatura de Ingeniería de Fabricación que se imparte en inglés. Los trabajos realizados también se han elaborado en lengua inglesa, 
destacándose con una participación del 100\% del alumnado inscrito en dicha asignatura, formando 4 grupos de trabajo y con una evaluación media de 8,5 puntos.

\section{DISCUSIÓN}

El seguimiento y evaluación del trabajo propuesto al alumnado ha contado con un total de 8 docentes, como se ha comentado con anterioridad. Esto ha implicado una participación muy importante de los miembros que integran el Área de Conocimiento de Ingeniería de los Procesos de Fabricación, lo que ha permitido crear un grupo de trabajo para establecer los objetivos, metodología y criterios de seguimiento y evaluación que han sido expuestos en el presente documento.

Esta experiencia docente ha facilitado homogeneizar criterios educativos a la hora de impartir la asignatura a un número de grupos tan numeroso, como es el considerado en este caso, reduciendo las variaciones que pudiera aportar cada docente de forma independiente.

Como se ha indicado, el grado de participación en la actividad propuesta ha sido muy elevado $(89,51 \%)$, donde han intervenido hasta 409 estudiantes. El hecho de que la evaluación del trabajo forme parte de la evaluación final de la asignatura, sin duda, ha aumentado el interés del alumnado en su desarrollo. Además, como puede observarse, por cada titulación académica, la participación está en parámetros similares, por lo que no se considera que pudiera haber surgido alguna incidencia a valorar durante el desarrollo de la actividad.

Esta elevada participación ha permitido elaborar hasta 85 términos diferentes relacionados con la Ingeniería de Fabricación, que suponen una herramienta educativa para posteriores cursos académicos que faciliten el aprendizaje al nuevo alumnado. Dichos vídeos se pueden utilizar durante el desarrollo del contenido en el aula, pero también para poner a disposición del alumnado a través de Campus Virtual u otro medio digital a fin de que puedan visualizarse durante el desarrollo del proceso de aprendizaje individual del alumnado.

Se han formado 111 grupos de trabajo, de entre 3 y 4 estudiantes, y cada uno de los integrantes ha formado parte en la elaboración del trabajo propuesto. Cada grupo se ha organizado en la distribución de tareas a realizar por cada integrante, así como en la puesta en común de las actividades realizadas, para alcanzar un consenso en el resultado final a presentar del trabajo, con el objeto de asegurar el trabajo en equipo. En el caso de que algún integrante no haya formado parte del trabajo, su evaluación ha sido de 0 puntos, siendo el resto de integrantes de grupo quienes han indicado al personal docente la no participación del alumno en cuestión.

La evaluación obtenida en los trabajos puede considerarse satisfactoria (8,02 puntos). Se observa que no hay elevadas variaciones en las evaluaciones de las diferentes titulaciones, por lo que puede considerarse que se han aplicado los criterios de evaluación de la misma forma por todos los docentes que han tutorizado el trabajo. Aunque la asignatura de Ingeniería de Fabricación es obligatoria en las cuatro titulaciones objeto de estudio, el mayor grado de relación de la asignatura con las titulaciones de Ingeniería Mecánica y Tecnologías Industriales ha podido facilitar el interés y dedicación por parte del alumnado en la elaboración de la actividad propuesta, generando por tanto un resultado de evaluación mejor que en el resto de titulaciones.

Los grados de Ingeniería Mecánica y de Ingeniería en Tecnologías Industriales han obtenido una mejor calificación que los otros dos grados, considerando además que en dichas dos titulaciones se han realizado un mayor número de trabajos.

Finalmente, el grupo de la titulación del Grado en Ingeniería de Tecnologías Industriales que se imparte en lengua inglesa ha obtenido una calificación ligeramente por encima de la media de todas las titulaciones, lo que significa que la lengua no ha supuesto una dificultad adicional a la hora de realizar la actividad por parte del alumnado.

\section{CONCLUSIONES}

En relación a los objetivos planteados inicialmente y a la vista de los resultados obtenidos en esta experiencia se puede considerar que:

Se ha generado una base de datos importante de vídeos de tipo educativo de corta duración, relacionados con la ingeniería de fabricación, que son una herramienta docente a utilizar en posteriores cursos académicos, facilitadora del proceso de aprendizaje.

La actividad realizada ha fomentado el trabajo colaborativo entre el alumnado evidenciado a partir de las sesiones de seguimiento del trabajo en el aula.

El alumnado ha utilizado TIC para realizar búsqueda de información relacionada con la Ingeniería de Procesos de Fabricación, así como diferentes softwares para realizar la edición de vídeos. 
Se han familiarizado con la elaboración de referencias bibliográficas en formato IEEE, propio en el ámbito de la ingeniería, que será un recurso a utilizar en futuros estudios de investigación que realicen, como puede ser el trabajo fin de grado.

Además, el alumnado ha presentado su trabajo al resto de estudiantes, fomentando la interrelación del alumnado, no solo con los integrantes de su grupo de trabajo, sino con el resto de estudiantes.

Por tanto, la implantación de esta actividad ha supuesto un elemento motivador y de aprendizaje en el uso de nuevas tecnologías de información y comunicación en el alumnado que desarrolla la asignatura, así como para el personal docente que forma parte del área de conocimiento en la cual se ha realizado un trabajo de forma coordinada, mejorando la actividad docente durante el curso académico en el que se ha desarrollado, así como facilitar nuevas metodologías educativas para futuros años.

\section{REFERENCIAS}

Ahmadi, S. (2011). Chapter 3 - \{IEEE 802.16m Reference Model and Protocol Structure. In Mobile WiMAX (pp. 6196).

Antoli, N. S. (2015). Metodologías participativas y Facebook en el ámbito universitario Participatory methodologies and Facebook in Higher Education. Innoeduca. International Journal of Technology and Educational Innovation, 1(1), 25-32.

Araya, R. (2017). Complejidad , fenómenos que emergen y cognición corporizada en educación Complexity , emergence and embodied cognition in education. Innoeduca. International Journal of Technology and Educational Innovation, 3(2), 159-170.

Area, M. (2005). A modo de introducción. Revista Electrónica de Investigación y Evaluación Educativa, 11(1), 3-25.

Arias Masa, J. (2008, January 18). Evaluación de la calidad de cursos virtuales: Indicadores de calidad y construcción de un cuestionario de medida. Aplicación al ámbito de asignaturas de ingeniería telemática. Universidad de Extremadura. Servicio de Publicaciones.

Ausín, V., Abella, V., Delgado, V. y Hortigüela, D. (2016). Aprendizaje Basado en Proyectos a través de las TIC: Una Experiencia de Innovación Docente desde las Aulas Universitarias. Formación Universitaria, 9(3), 31-38.

Barroso Osuna, J., Cabero Almenara, J. y Moreno Fernández, A. M. (2016). La utilización de objetos de aprendizaje en Realidad Aumentada en la enseñanza de la medicina. Innoeduca. International Journal of Technology and Educational Innovation, 2(2), 77.

Bustamante, J. C., Rábanos, N. L., Vicente Sánchez, E., Sánchez, J. C., Luis, J., Laborda, A. y Salavera, C. (2016). El uso de las píldoras formativas competenciales como experiencia de innovación docente en el grado de magisterio en educación infantil. Reidocrea, 5(22), 223 234.

Cardona, P. y Wilkinson, H. (2006). Trabajo En Equipo. IESE Business School, 3, 1-8.

Cazorla, M. D. C. (2011). Una aproximación a los aspectos positivos y negativos derivados de la puesta en marcha del Plan Bolonia en la Universidad Española. REJIE: Revista Jurídica de Investigacion e Innovación Educativa, 4, 93-104.

Guerra, S., González, N. y García, R. (2010). Utilización de las TIC por el profesorado universitario como recurso didáctico. Comunicar, XVIII(35), 141-148.

Hernández, J.P., Torrijos, P. (2019). Percepción del profesorado universitario sobre integración de las Tecnologías de la información y la Comunicación (TIC) en las modalidades docentes. Influencia del género y la edad. Edmetic. REvista de Educación Mediática y TIC, 8 (1), 128-146.

Maquilón Sánchez, J. J., Mirete Ruiz, A. B., García Sánchez, F. A. y Hernández Pina, F. (2013). Valoración de las TIC por los estudiantes universitarios y su relación con los enfoques de aprendizaje. Revista de Investigación Educativa, 31(2), 537-554.

Mayer, R. E. (2005). The Cambridge handbook of multimedia learning. Cambridge University Press.

Monedero, R., Higueras, A. C. y Luengo, J. T. (2017). Vídeotutoriales y códigos QR: recursos TIC en laboratorios de Ciencias de la Comunicación audiovisual labs. Innoeduca. International Journal of Technology and Educational Innovation, 3, 137-145.

Morales, M., Trujillo, J.M., Raso, F. (2015). Percepciones acerca de la integración de las TIC en el proceso de enseñanza-aprendizaje de la Universidad. Pixel-Bit. Revista de Medios y Educación, 46, 103-117

Oswaldo, M. y Gamonal, C. (2016). Lectura y escritura con recursos TIC en Educación Superior. Evaluación de la competencia digital. International Journal of Technology and Educational Innovation, 2(1), 64-73. doi:10.20548/innoeduca.2016.v2i1.1046

PrietoDíaz, V., Quiñones La Rosa, I., Ramírez Durán, G., Fuentes Gil, Z., Labrada Pavón, T., Pérez Hechavarría, O. y Montero Valdés, M. (2011). Impacto de las tecnologías de la información y las comunicaciones en la educación y nuevos paradigmas del enfoque educativo. Revista Cubana de Educacion Medica Superior., 25(1), 95-102.

Quesadas Sánchez, M. J. (2014). Creación de vídeos educativos como estrategia didáctica para la formación de futuros docentes de inglés. Revista Electrónica "Actualidades Investigativas En Educación”, 15, 1-19. doi:10.15517/aie.v15i1.17588

Río, A. B. F. del y Arias, A. J. B. (2015). El cortometraje como herramienta innovadora para el alumnado con altas capacidades en Educación Primaria. Innoeduca. International Journal of Technology and Educational Innovation, 3(1), 28-36.

Ros Gálvez, A. y Rosa García, A. (2014). Uso del vídeo docente para la clase invertida: evaluación, ventajas e inconvenientes, 423-441.

Serrat, N. y Jarauta, B. (2010). Active and Collaborative Methodologies to Help Improve the Learning of Future Pedagogues. Journal of Teaching and Learning in Higher Education, 3(1), 67-69.

Tello Díaz, J. y Aguaded Gomez, J. I. (2009). Desarrollo profesional docente ante los nuevos retos de las tecnologías de la información y la comunicación en los centros educativos. Pixel-Bit. Revista de Medios y Educación, 34, 31-47. 